\title{
UNFRACTIONATED HEPARIN AND MECHANICAL THROMBOPROPHYLAXIS IN HIP ARTHROPLASTY
}

\author{
Flávio Luis Garcia ${ }^{1}$, Murilo Humberto Tobias Marins ${ }^{2}$, Thiago Bortoletto Raddi ${ }^{2}$, Celso Hermínio Ferraz Picado ${ }^{1}$
}

\begin{abstract}
Objective: To evaluate the efficacy and safety of unfractionated heparin associated with mechanical prophylaxis as a method for preventing venous thromboembolism in hip arthroplasty. Method: We retrospectively reviewed the records of 181 hip arthroplasties out of 216 consecutive cases performed over a period of 39 months in our hospital. We excluded 35 cases due to non-adherence to the standardized method of thromboprophylaxis or loss to follow-up. All arthroplasties evaluated completed one-year follow-up after surgery with five consultations at predefined periods. Efficacy of the proposed method was evaluated by the occurrence of symptomatic venous thromboembolism confirmed by specific tests and safety was determined by the lack of occurrence of major bleeding according to criteria established by the
\end{abstract}

International Society on Thrombosis and Haemostasis. Results: There were four cases of symptomatic venous thromboembolism (2.2\%), with three cases of deep vein thrombosis $(1.65 \%)$ and one case of pulmonary embolism $(0.55 \%)$. We found one case of severe bleeding (0.55\%). Conclusion: Unfractionated heparin associated with mechanical prophylaxis proved to be an effective and safe method for preventing venous thromboembolism in patients undergoing hip arthroplasty, presenting rates of thromboembolic complications and major bleeding within the range reported with other methods currently used of thromboprophylaxis.

\section{Level of Evidence IV, Case Series}

Keywords: Venous thrombosis. Pulmonary embolism. Arthroplasty, replacement, hip. Hemorrhage. Heparin.

\section{INTRODUCTION}

Venous thromboembolism (VTE) comprises deep vein thrombosis (DVT) and pulmonary thromboembolism (PTE), and it is one of the most serious complications associated with hip arthroplasty and a major cause of death during the first three months after surgery. ${ }^{1}$ In patients undergoing hip arthroplasty, prophylaxis of VTE has been standard practice for decades, with proven reduction in the incidence and mortality associated with this complication. ${ }^{2,3}$

The current options for the drug prophylaxis of VTE in hip arthroplasty are numerous, such as aspirin, unfractionated heparin, low molecular weight heparins, vitamin $\mathrm{K}$ antagonists, direct thrombin inhibitors and direct inhibitors of activated $X$ factor, all with grade of recommendation $1 \mathrm{~B}$ according to the most recent guidelines of the American College of Chest Physicians (ACCP). ${ }^{4}$ Despite the consensus that VTE prophylaxis is necessary, ${ }^{3}$ the ideal prophylactic regimen is still undefined, being a delicate balance between efficiency and security: insufficient anticoagulation exposes patients to the risk of VTE while ex- cessive anticoagulation can lead to major bleeding, hematoma at the surgical site, reoperation and infection, all with potential deleterious effect on the final outcome.

The objective of this study was to evaluate the efficacy and safety of thromboprophylaxis standard scheme for hip arthroplasty in our hospital, combining drug prophylaxis through unfractionated heparin along with mechanical prophylaxis through an intermittent pneumatic compression device for lower limbs and elastic stockings.

\section{MATERIALS AND METHODS}

The study was approved by the Research Ethics Committee of our institution (Instrument № 549 810). This is a retrospective study of a consecutive case series type. All patients undergoing total or partial primary hip arthroplasty and hip arthroplasty revision from September 2009 to December 2012 in our hospital were included. Exclusion criteria were failure to attend all five routine consultations scheduled during the first year after surgery (two weeks, one month, two months, six months and one year

All the authors declare that there is no potential conflict of interest referring to this article.

1. Universidade de São Paulo (FMRP-USP), Faculdade de Medicina de Ribeirão Preto, Ribeirão Preto, SP, Brazil.

2.Universidade de São Paulo (HCFMRP-USP), Hospital das Clínicas da Faculdade de Medicina de Ribeirão Preto, Ribeirão Preto, SP, Brazil.

Work developed at Universidade de São Paulo, Hospital das Clínicas da Faculdade de Medicina de Ribeirão Preto, Ribeirão Preto, SP, Brazil. Correspondence: Avenida Bandeirantes, 3900, Bairro Monte Alegre, 14048-900 Ribeirão Preto, SP, Brazil. flavio@fmrp.usp.br 
after surgery) or non-compliance with the standard thromboprophylaxis scheme, which was conducted using 5,000 IU of unfractionated heparin subcutaneously every 12 hours, starting four to six hours after surgery and continued until the $30^{\text {th }}$ day after surgery, combined with the use of an intermittent pneumatic compression device for the lower limbs (Angiotron 1 Plus $^{\circledR}$, Angiotronic SA, São Paulo, Brazil) at $40 \mathrm{mmHg}$ for 40 minutes, three times a day during the hospital stay (Figure 1) and replaced after discharge by $7 / 8$ medium compression elastic stockings $(20-30 \mathrm{mmHg})$ until the $30^{\text {th }}$ day after surgery.

All patients were operated through the direct lateral access. ${ }^{5}$ The type of anesthesia was defined by the responsible anesthesiologist individually for each patient. As a routine postoperative rehabilitation, patients started isometric quadriceps exercises and active flexion-extension of the ankle on the same day of surgery and triple flexion active exercises (hip, knee and ankle) on the next day after surgery. We use intravenous antibiotic prophylaxis with $2 \mathrm{~g}$ cefazolin in the anesthetic induction and $1 \mathrm{~g}$ every eight hours for two days. A $3.2 \mathrm{~mm}$ aspirating drain was used in all patients and removed the day after the surgery. Gait began in the second or third day after surgery with the aid of a walker. Hospital discharge normally occurred between the third and the fifth day after surgery. As already mentioned, patients returned to routine follow up visits at two weeks, a month, two months, six months and one year after surgery.

During the study period 162 total arthroplasties, 32 partial arthroplasties and 22 arthroplasty revisions were made in 215 patients (one patient with total bilateral primary hip arthroplasty with an eight months interval between surgeries). We exclude from the study 35 patients (13 patients with total hip replacement, 19 patients with partial arthroplasty and three patients with revision arthroplasty); four were excluded due to non-adherence to the standard scheme of thromboprophylaxis and 31 due to non attendance to scheduled returns (loss of follow-up); we found out that four of these 31 patients lost to follow-up died in our hospital before completing one year after surgery for causes unrelated to VTE (three sepsis due to pneumonia and one by cardiogenic shock due to cardiac arrhythmia), but in the other 27 cases we could not determine the reason for the loss of follow-up. The main demographic and surgical data of the assessed patients and excluded patients are listed in Table 1. None of the 180 patients evaluated (181 arthroplasties) was convened specifically for this study; all data were obtained from medical records of our hospital.

The parameter for evaluating the effectiveness of our thromboprophylaxis scheme was the occurrence of symptomatic VTE (DVT or PTE). In cases where there was a clinical suspicion of VTE this hypothesis should be confirmed objectively by Doppler ultrasound (in suspected DVT) or chest computed tomography angiography (in suspected nonfatal PTE) or necropsy (in suspected fatal PTE). The parameter for assessing the safety of the proposed thromboprophylaxis scheme was the occurrence of major bleeding, according to the criteria proposed by the International Society on Thrombosis and Haemostasis (ISTH), ${ }^{6}$ defined as a bleeding with a fatal outcome or critical location (intracranial , intraocular, retroperitoneal or spinal cord) or which required reoperation for its control or postoperative bleeding that required transfusion of two or more red blood cell units. The confidence intervals of $95 \%(\mathrm{Cl} 95 \%)$ to the incidences

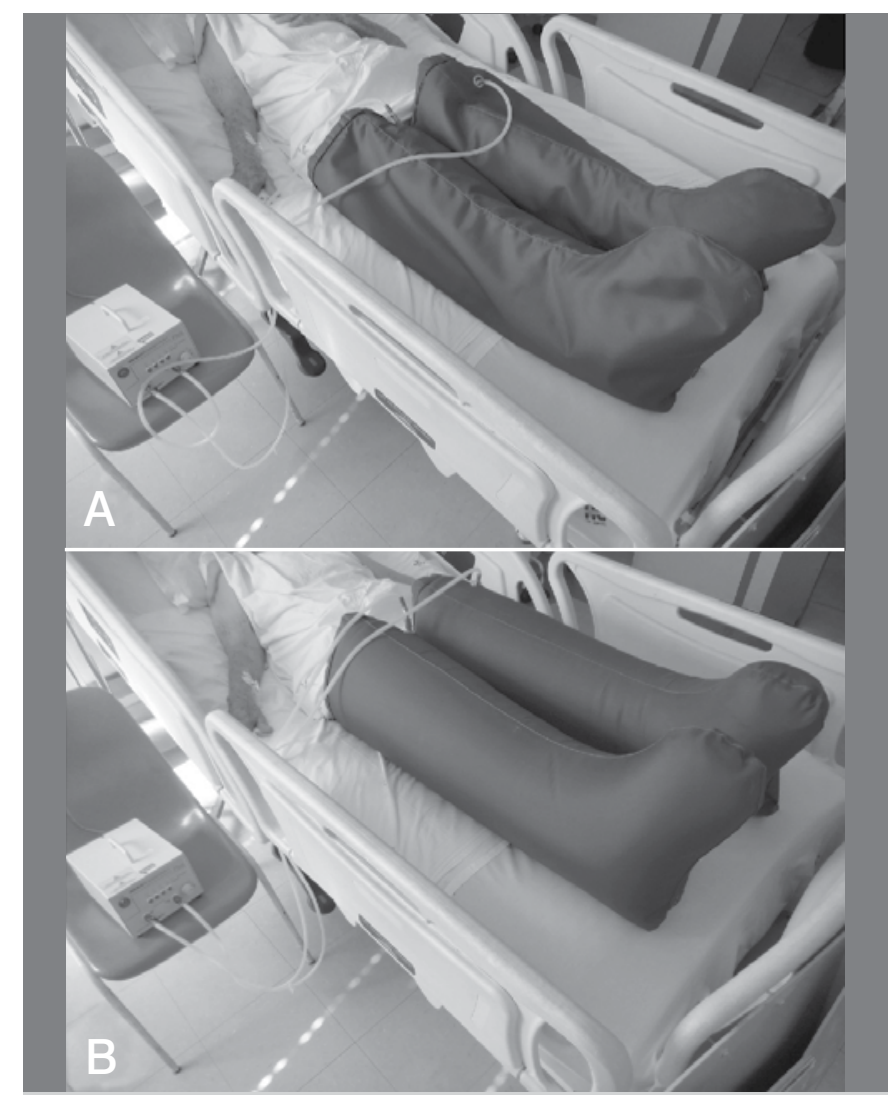

Figure 1. Intermittent pneumatic compression device for the lower limbs being used on a patient who underwent hip arthroplasty on the emptying phase $(A)$ and the filling phase $(B)$; the device compressor lies on the chair next to the patient.

found for VTE and major bleeding were calculated with the StatsDirect ${ }^{\circledR}$ software (StatsDirect Limited, Altrincham, England).

\section{RESULTS}

We found four patients with symptomatic VTE and one patient with major bleeding in the 181 evaluated arthroplasties.

One of the patients who presented symptomatic VTE was male, 85 years old with femoral neck fracture treated with bipolar hemiarthroplasty and presented fatal PTE on the third day after surgery. Another case of VTE occurred in a male patient, 48 years old, subjected to total hybrid arthroplasty for osteoarthritis secondary to Perthes disease who presented with symptomatic DVT and partial obstruction of the common femoral vein in the sixth postoperative day, evolving to complete resolution of the symptoms with drug treatment. Finally, two other cases of VTE occurred in patients undergoing hip replacement revision, with partial thrombosis of the common femoral vein in both; one patient was diagnosed on the tenth day after surgery, she was a female, 76 years old and the other was male, 86 years old, and was diagnosed on the second day after surgery. Both also evolved to complete resolution of symptoms with drug treatment. Thus, in 181 assessed arthroplasties evaluated, we found a total of four thromboembolic events $(2.2 \%, 95 \% \mathrm{Cl}$, range $0.61 \%$ to $5.56 \%)$, three cases of DVT $(1.65 \%, \mathrm{Cl} 95 \%$, range $0.34 \%$ to $4.77 \%)$ and one case of PTE $(0.55 \%, 95 \% \mathrm{Cl}$, range $0.01 \%$ to $3.04 \%$ ). 
The only occurrence of major bleeding was observed in a female patient, 73 years old, who had received a cemented total hip replacement due to femoral neck fracture and developed hematoma at the surgical site that required reoperation (drainage) on the fifth day after arthroplasty surgery. The incidence of major bleeding was, therefore, $0.55 \%$ (95\% $\mathrm{Cl}$ : $0.01 \%$ to $3.04 \%)$. These data are summarized in Table 2.

Table 1. Demographic and surgical characteristics of patients.

\begin{tabular}{|c|c|c|c|}
\hline Characteristics & All & Assessed & Excluded \\
\hline Age range in years old (mean) & $30-93(63.2)$ & $30-93(61.2)$ & $40-91(71.5)$ \\
\hline Gender & $113 \mathrm{~m} / 102 \mathrm{f}$ & $96 \mathrm{~m} / 84 \mathrm{f}$ & $17 \mathrm{~m} / 18 \mathrm{f}$ \\
\hline \multicolumn{4}{|l|}{ Indication of primary arthroplasty } \\
\hline Primary osteoarthritis & 90 & 85 & 5 \\
\hline Fracture of the femoral neck & 48 & 27 & 21 \\
\hline ONFH & 22 & 19 & 3 \\
\hline Sequelae of trauma & 19 & 16 & 3 \\
\hline Rheumatic disease & 5 & 5 & 0 \\
\hline Sequelae of Perthes disease & 4 & 4 & 0 \\
\hline Sequelae of epiphysiolysis & 3 & 3 & 0 \\
\hline $\mathrm{DDH}$ & 2 & 2 & 0 \\
\hline Neoplasia & 1 & 1 & 0 \\
\hline \multicolumn{4}{|l|}{ Type of primary arthroplasty } \\
\hline Total non-cemented & 79 & 74 & 5 \\
\hline Total hybrid & 63 & 57 & 6 \\
\hline Total cemented & 20 & 18 & 2 \\
\hline Partial bipolar cemented & 26 & 11 & 15 \\
\hline Partial unipolar cemented & 6 & 2 & 4 \\
\hline \multicolumn{4}{|l|}{$\begin{array}{l}\text { Indication of revision } \\
\text { arthroplasty }\end{array}$} \\
\hline Loosening & 12 & 10 & 2 \\
\hline Periprosthetic fracture & 5 & 4 & 1 \\
\hline Polyethylene tear & 5 & 5 & 0 \\
\hline \multicolumn{4}{|l|}{$\begin{array}{c}\text { Component substituted in } \\
\text { revision }\end{array}$} \\
\hline Only femoral & 5 & 4 & 1 \\
\hline Only acetabular & 6 & 4 & 2 \\
\hline Both & 6 & 6 & 0 \\
\hline Only polyethylene and head & 5 & 5 & 0 \\
\hline Number of patients & 215 & 180 & 35 \\
\hline Number of arthroplasties & 216 & 181 & 35 \\
\hline
\end{tabular}

m: male; f: female; ONFH: osteonecrosis of the femoral head; DDH: developmental dysplasia of the hip.

Table 2. Incidence of thromboembolic events and severe bleeding

\begin{tabular}{c|c}
\hline Total of assessed arthroplasties & 181 \\
\hline VTE (\%) [IC 95\%] & $4(2.20 \%)[0.61 \%-5.56 \%]$ \\
\hline DVT & $3(1.65 \%)[0.34 \%-4.77 \%]$ \\
\hline PTE & $1(0.55 \%)[0.01 \%-3.04 \%]$ \\
\hline Severe bleeding (\%) [IC 95\%] & $1(0.55 \%)[0.01 \%-3.04]$ \\
\hline
\end{tabular}

VTE: venous thromboembolism; DVT: deep venous thrombosis; PTE: pulmonary thromboembolism.

\section{DISCUSSION}

The number of hip arthroplasties performed worldwide demonstrates a sustained increase ${ }^{7,8}$ and the prevention of VTE in these patients is of utmost importance. In the absence of thromboprophylaxis measures, screening studies in patients undergoing hip arthroplasty showed incidences of DVT and PTE that can reach $42-60 \%$ and $1-21 \%$ of cases, respectively. ${ }^{9-12} \mathrm{Admittedly}$ one yet undefined proportion of these thromboembolic events are completely asymptomatic and evolve to spontaneous resolution, ${ }^{13,14}$ however, prophylaxis of VTE either through mechanical methods and/or with medicines, is recommended for patients undergoing hip arthroplasty for proven reduction of the incidence and mortality associated with thromboembolic complications. ${ }^{2,3}$

The arsenal of drugs available for VTE prophylaxis in patients undergoing hip arthroplasty includes aspirin, unfractionated heparin, low molecular weight heparin (enoxaparin, dalteparin, nadroparin), vitamin $\mathrm{K}$ antagonists (warfarin, phenprocoumon) and more recently, the so-called new oral anticoagulants, that can be divided into direct inhibitors of activated $X$ factor (apixabana, rivaroxaban) and the direct thrombin inhibitor (dabigatran). Unfortunately, even after years of use in clinical practice and hundreds of published studies, the ideal method of thromboprophylaxis is still undefined; and even conflicting recommendations occur in the guidelines proposed by different scientific societies related to the topic., ${ }^{9,15}$

Our routine method in preventing VTE in hip replacement lasts 30 days and involves mechanical prophylaxis through an intermittent pneumatic compression device while the patient is hospitalized, being replaced by elastic stockings from the time of hospital discharge and chemoprophylaxis with unfractionated heparin at a dose of 5,000 IU subcutaneously every $12 \mathrm{~h}$ for 30 days, starting four to six hours after surgery. Unfractionated Heparin is a heterogeneous mixture of polysaccharide molecules which bind to antithrombin, forming a complex which inhibits the action of the activated $X$ factor and thrombin, thereby preventing conversion of fibrinogen to fibrin. Our rationale for choosing this drug lies in the fact that heparin is a widely available low cost drug, (including being made freely available by the Unified Health System), does not require monitoring of blood coagulation parameters when used in prophylactic treatment and by the fact that it has a specific antidote, protamine, if necessary. Its only drawback, in our view, is the parenteral route of administration (subcutaneous) in two daily doses. Our study has several limitations, as the small number of cases evaluated; the absence of a control group; the fact that this is a retrospective study, where we rely on the accuracy of the information contained in the medical records; and finally the loss of follow-up of 31 patients, among which we cannot exclude the possibility of other cases of VTE, which would modify the results presented here.

On the other hand, our study presents as strength the absence of a specific selection of patients among those undergoing hip arthroplasty in our hospital, a significant fact taking into consideration that in randomized controlled trials evaluating the efficacy and safety of new medicines for thromboprophylaxis, patients presenting a clinical condition with high risk of VTE (thrombophilia, previous VTE, cancer, etc.) or at high risk of bleeding (liver diseases, recent use of antiplatelet agents, etc.) 
are systematically excluded from recruitment. ${ }^{9}$ Therefore, our study was able to assess in a "real life" condition the efficacy and safety of a thromboprophylaxis scheme routinely used by our team. Another aspect of our study that should be highlighted is the follow-up of patients for over a year after surgery; the importance of this detail is the fact that most studies on the incidence of VTE in hip arthroplasty terminate the evaluation of patients between six and twelve weeks postoperatively, which can lead to underestimated rates of VTE, as it is shown that incidence of thromboembolic events may remain elevated for up to one year after arthroplasty. ${ }^{16,17}$

Our overall incidence of symptomatic thromboembolic events (2.2\%) and our specific incidence of DVT (1.65\%) and PTE (0.55\%) are within the rates reported in the literature using different drugs for thrombosis prophylaxis, ${ }^{16-21}$ ranging between $0.5-4.6 \%, 0.3$ - $3.4 \%$ and $0.2-1.7 \%$, respectively.

We also found a major bleeding incidence $(0.55 \%)$ which is within the levels reported with the use of different drugs $(0.4-7 \%)^{22,23}$ We consider important to emphasize that recent studies ${ }^{23-25}$ demonstrated a greater tendency to haemorrhagic complications and prolonged blood drainage through the surgical wound with new oral anticoagulants; although further clinical studies are needed to clarify this issue, this should remind us of the importance of moderation and caution before the immediate adoption of new drugs to replace those most widely studied and used in clinical practice.

\section{CONCLUSION}

Unfractionated heparin associated to mechanical prophylaxis proved to be an effective and safe method in preventing deep vein thrombosis in patients undergoing hip replacement, presenting rates of thromboembolic complications and major bleeding within the limits reported with other current methods of thromboprophylaxis.

\section{REFERENCES}

1. Berstock JR, Beswick AD, Lenguerrand E, Whitehouse MR, Blom AW. Mortality after total hip replacement surgery. Bone Joint Res. 2014;3(6):175-82.

2. 2. Dahl OE, Caprini JA, Colwell CW Jr, Frostick SP, Haas S, Hull RD, et al. Fatal vascular outcomes following major orthopedic surgery. Thromb Haemost. 2005;93(5):860-6.

3. Lieberman JR, Pensak MJ. Prevention of venous thromboembolic disease after total hip and knee arthroplasty. J Bone Joint Surg Am. 2013;95(19):1801-11.

4. Falck-Ytter Y, Francis CW, Johanson NA, Curley C, Dahl OE, Schulman S, et al. Prevention of VTE in orthopedic surgery patients: antithrombotic therapy and prevention of thrombosis, $9^{\text {th }}$ ed: American College of Chest Physicians evidence-based clinical practice guidelines. Chest. 2012;141(2 Suppl):e278S-325S.

5. Hardinge K. The direct lateral approach to the hip. J Bone Joint Surg Br. 1982;64(1):17-9.

6. Schulman S, Angerås U, Bergqvist D, Eriksson B, Lassen MR, Fisher W. Definition of major bleeding in clinical investigations of antihemostatic medicinal products in surgical patients. J Thromb Haemost. 2010;8(1):202-4.

7. Pivec R, Johnson AJ, Mears SC, Mont MA. Hip arhroplasty. Lancet. 2012;380(9855):1768-77.

8. Pabinger $\mathrm{C}$, Geissler A. Utilization rates of hip arthroplasty in OECD countries Osteoarthritis Cartilage. 2014;22(6):734-41.

9. Chari A, Khokhar A, Murray D, McNally M, Pandit H. Venous thromboembolism and its prophylaxis in elective total hip arthroplasty: an international perspective. Hip Int. 2012;22(1):1-8.

10. Arcelus JI, Kudrna JC, Caprini JA. Venous thromboembolism following major orthopedic surgery: what is the risk after discharge? Orthopedics. 2006;29(6):506-16.

11. Warwick D, Williams MH, Bannister GC. Death and thromboembolic disease after total hip replacement. A series of 1162 cases with no routine chemical prophylaxis. J Bone Joint Surg Br. 1995;77(1):6-10.

12. Eriksson BI, Kälebo $\mathrm{P}$, Anthymyr BA, Wadenvik H, Tengborn L, Risberg $B$ Prevention of deep-vein thrombosis and pulmonary embolism after total hip replacement. Comparison of low-molecular-weight heparin and unfractionated heparin. J Bone Joint Surg Am. 1991;73(4):484-93.

13. Tzoran I, Saharov G, Brenner B, Delsart D, Román P, Visoná A, et al. Silent pulmonary embolism in patients with proximal deep vein thrombosis in the lower limbs. J Thromb Haemost. 2012;10(4):564-71.
14. Kearon C. Natural history of venous thromboembolism. Circulation. 2003;107(23 Suppl 1):I22-30.

15. Barrack RL. Current guidelines for total joint VTE prophylaxis: dawn of a new day. J Bone Joint Surg Br. 2012;94(11 Suppl A):3-7.

16. Dahl OE, Gudmundsen TE, Pripp AH, Aanesen JJ. Clinical venous thromboembolism following joint surgery: effect of extended thromboprophylaxis on its annual frequency and postoperative pattern over 22 years. Clin Appl Thromb Hemost. 2014;20(2):117-23.

17. Pedersen AB, Johnsen SP, Sørensen HT. Increased one-year risk of symptomatic venous thromboembolism following total hip replacement: a nationwide cohort study. J Bone Joint Surg Br. 2012;94(12):1598-603.

18. Quinlan DJ, Eriksson BI. Novel oral anticoagulants for thromboprophylaxis after orthopaedic surgery. Best Pract Res Clin Haematol. 2013;26(2):171-82.

19. Lapidus LJ, Ponzer S, Pettersson H, de Bri E. Symptomatic venous thromboembolism and mortality in orthopaedic surgery: an observational study of 45.968 consecutive procedures. BMC Musculoskelet Disord. 2013;14(1):177

20. Januel JM, Chen G, Ruffieux C, Quan H, Douketis JD, Crowther MA, et al. Symptomatic in-hospital deep vein thrombosis and pulmonary embolism following hip and knee arthroplasty among patients receiving recommended prophylaxis: a systematic review. JAMA. 2012;307(3):294-303.

21. Phillips CB, Barrett JA, Losina E, Mahomed NN, Lingard EA, Guadagnoli E, et al. Incidence rates of dislocation, pulmonary embolism, and deep infection during the first six months after elective total hip replacement. J Bone Joint Surg Am. 2003;85-A(1):20-6.

22. Mortazavi SM, Hansen P, Zmistowski B, Kane PW, Restrepo C, Parvizi J. Hematoma following primary total hip arthroplasty: a grave complication. $J$ Arthroplasty. 2013;28(3):498-503.

23. Gill SK, Theodorides A, Smith N, Maguire E, Whitehouse SL, Rigby MC, et al. Wound problems following hip arthroplasty before and after the introduction of a direct thrombin inhibitor for thromboprophylaxis. Hip Int. 2011;21(6):678-83.

24. Sindali K, Rose B, Soueid H, Jeer P, Saran D, Shrivastava R. Elective hip and knee arthroplasty and the effect of rivaroxaban and enoxaparin thromboprophylaxis on wound healing. Eur J Orthop Surg Traumatol. 2013;23(4):481-6.

25. Bloch BV, Patel V, Best AJ. Thromboprophylaxis with dabigatran leads to an increased incidence of wound leakage and an increased length of stay after total joint replacement. Bone Joint J. 2014;96-B(1):122-6. 